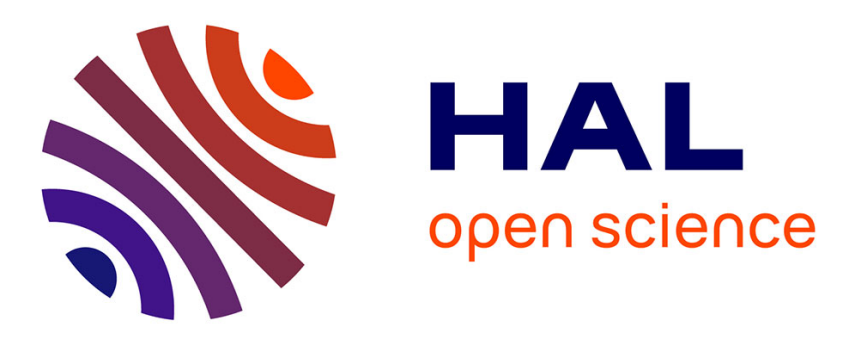

\title{
Wideband discrete transformation of acoustic signals in underwater environments
}

Nicolas Josso, Jun J. Zhang, Antonia Papandreou-Suppappola, Cornel Ioana, Jerome I. Mars, Cedric Gervaise, Yann Stéphan

\section{To cite this version:}

Nicolas Josso, Jun J. Zhang, Antonia Papandreou-Suppappola, Cornel Ioana, Jerome I. Mars, et al.. Wideband discrete transformation of acoustic signals in underwater environments. ACSSC 2007 - 43rd Annual Asilomar Conference on Signals Systems and Computers, Nov 2009, Pacific Grove, Californie, United States. pp.118 - 122, 10.1109/ACSSC.2009.5470155 . hal-00444394

\author{
HAL Id: hal-00444394 \\ https://hal.science/hal-00444394
}

Submitted on 8 Jan 2010

HAL is a multi-disciplinary open access archive for the deposit and dissemination of scientific research documents, whether they are published or not. The documents may come from teaching and research institutions in France or abroad, or from public or private research centers.
L'archive ouverte pluridisciplinaire $\mathbf{H A L}$, est destinée au dépôt et à la diffusion de documents scientifiques de niveau recherche, publiés ou non, émanant des établissements d'enseignement et de recherche français ou étrangers, des laboratoires publics ou privés. 


\title{
Wideband Discrete Transformation of Acoustic Signals in Underwater Environments
}

\author{
Nicolas F. Josso*, Jun Jason Zhang ${ }^{\dagger}$, Antonia Papandreou-Suppappola ${ }^{\dagger}$, Cornel Ioana*, \\ Jerome I. Mars*, Cédric Gervaise ${ }^{\ddagger}$, and Yann Stéphan ${ }^{\S}$ \\ *GIPSA-lab /DIS, Grenoble Institute of Technology, GIT, Grenoble, France \\ ${ }^{\dagger}$ School of Electrical, Computer and Energy Engineering, Arizona State University, Tempe, AZ, USA \\ ${ }^{\ddagger}$ E3I2, EA3876, ENSIETA, Université Européenne de Bretagne, 29806 Brest Cedex, France

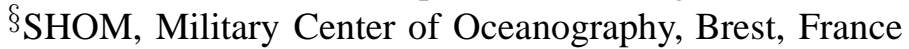 \\ E-mails: nicolas.josso@gipsa-lab.inpg.fr, Jun.Zhang.EE@asu.edu \\ papandreou@asu.edu, cornel.ioana@gipsa-lab.inpg.fr
}

\begin{abstract}
We propose the use of discrete time-scale transformations of acoustic signals to characterize the wideband effect of underwater environment propagation. The representation depends on the Mellin transform, and it can be used to efficiently process the effect of the underwater environment. For sparse environments, we also consider a new approach with reduced computational complexity. The approach is based on a warping lagDoppler filtering technique in the wideband ambiguity function plane to separate ray paths and estimate their parameters. We validate the signal representation and filtering approach using real experimental data from the BASE07 experiment.
\end{abstract}

\section{INTRODUCTION}

The characterization of underwater acoustic signals in terms of propagation medium attributes is essential for a large number of applications, including underwater communications, geoacoustic inversion, sonar, and marine mammal monitoring. The time-varying nature of the environment and the relative motion between the transmitter-channel-receiver configuration most often introduce undesirable distortions such as multipath and Doppler on the transmitted signal. When a narrowband signal undergoes a Doppler scale change, the transformation can be approximated by a frequency shift as the signal bandwidth is much smaller than its central frequency. However, for underwater acoustic signals, this assumption does not hold because the signal bandwidth is comparable to or larger than the central frequency. A signal is considered wideband when the ratio of the bandwidth and the central frequency of the signal is

This work was partly supported by DGA (Délégation Générale pour l'Armement) under SHOM research grant N07CR0001 and by the US DoD MURI Grant No. AFOSR FA9550-05-1-0443 higher than 0.1 [1]. As a result, most underwater acoustic signals in the medium-to-high frequency region (500$10,000 \mathrm{~Hz}$ ) should be characterized by time delays and Doppler scale changes [2].

The scale changes are physical transformations that are the result of fast relative motion between the propagation medium or object and scatterers present in the environment. These physical changes can be characterized by the wideband spreading function (WSF) representation [1], [3], [4]. Specifically, the output of a wideband system can be represented as a continuous linear superposition of time shifts and Doppler scale changes on the input signal, weighted by the WSF. Based on the continuous WSF representation, an equivalent discrete time-scale signal characterization was developed in [5], [6] for processing purposes. This discrete channel characterization decomposes a transformed wideband signal into a sum of time-delayed and Doppler-scaled versions of the input signal, weighted by a smoothed and sampled version of the WSF.

Although this discrete time-scale signal representation is appropriate to use for underwater wideband channels, it can also be computationally intensive. If the channel is sparse, then propagating rays can arrive in groups of similar time-shift and scale components due to physical propagation constraints [2], [7]. Hence, in order to minimize the processing computational expense at the receiver, we propose to first detect and separate the various ray groups using the wideband ambiguity function (WAF) and a time-warping tool. The discrete time-scale model is then applied to each ray group to estimate the distinct transformation.

The paper is organized as follows. Section II provides the WSF channel characterization. In Section III, we assume channel sparsity, and we describe ray group 
detection and separation. The application of the proposed signal characterization to real data from the BASE07 experiment is presented in Section IV.

\section{Channel CHARACTERIZATION}

For most underwater acoustic applications, the propagating signal is considered to have wideband properties. This is due to the fact that the transmitted signal undergoes distortions such as multipath and Doppler scale changes when propagating in the underwater environment. The output $x(t)$ of the wideband underwater environment can be modeled as a superposition of timeshifted and Doppler-scaled versions of the input signal $s(t)$ as [5], [6]

$$
x(t)=\int_{0}^{T_{s}} \int_{\eta_{\min }}^{\eta_{\max }} \mathcal{X}(\tau, \eta) \sqrt{\eta} s(\eta(t-\tau)) d \eta d \tau,
$$

where $\tau$ is the time shift and $\eta$ is the scale parameter. The wideband spreading function (WSF), $\mathcal{X}(\tau, \eta)$, represents the strength of underwater scatterers for varying $\tau$ and $\eta$. Due to the physical properties of realistic underwater environments, we assume that the WSF is supported in the regions $\tau \in\left[0, T_{s}\right]$ and $\eta \in\left[\eta_{\min }, \eta_{\max }\right]$, where $T_{s}$ is the time delay spread of the channel and $\left[\eta_{\min }, \eta_{\max }\right]$ represent the range of possible scaling factors.

By geometrically sampling the scaling factors using the proprieties of the Mellin transform, it is possible to represent the environment in terms of discretized transformed parameters. The Mellin transform of a signal $s(t)$ is defined as

$$
M_{s}(\beta)=\int_{0}^{\infty}(1 / \sqrt{t}) s(t) e^{j 2 \pi \beta \ln \left(t / t_{r}\right)} d t,
$$

where $\beta \in \Re$ is the Mellin variable and $t_{r}>0$ is a normalization constant. Assuming that our signal is localized in the time-frequency domain, it can be shown that it is also localized in the Mellin domain [5], [8]. For a signal whose Mellin transform is bounded in $\beta \in\left[-\beta_{0} / 2, \beta_{0} / 2\right]$, the scaling factors in (1) can be geometrically sampled as $\eta=\eta_{0}^{m}$ where $m$ is an integer and $\eta_{0}=e^{1 / \beta_{0}}$. The time-delay can be uniformly sampled for each given scaling factor $\eta_{0}^{m}$ as $\tau=n /\left(\eta_{0}^{m} W\right)$ for the $n$th time-delay, where $W$ is the bandwidth of $s(t)$. As a result, the discrete time-scale representation of the system is given by [5]

$$
x(t)=\sum_{m=M_{0}}^{M_{1}} \sum_{n=0}^{N(m)} \Psi_{n, m} \eta_{0}^{m / 2} s\left(\eta_{0}^{m} t-n / W\right),
$$

where $\Psi_{n, m}$ is a smoothed and sampled version of the WSF [5], $M_{0}=\ln \left(\eta_{\min }\right) / \ln \left(\eta_{0}\right), M_{1}=$ $\ln \left(\eta_{\max }\right) / \ln \left(\eta_{0}\right), N(m)=\left\lceil\eta_{0}^{m} W T_{s}\right\rceil$, and $\lceil\cdot\rceil$ denotes the next larger integer. It is worth noting that the number of time-shift parameters is not the same for each scaling factor as $N$ is a function of $m$ in (3).

\section{WIDEBAND LAG-DOPPLER FILTERING METHOD}

\section{A. Ray Theory}

When the channel is sparse, we expect of lot of the discrete WSF values $\Psi_{n, m}$ in Equation (3) to be zero. As a result, it would be computationally intensive to try and estimate all the WSF values of a sparse underwater channel. Following ray theory, propagating rays of sparse channels can arrive in groups of similar time delay and scale components due to the channel's physical propagation properties [2], [7]. It can actually be shown that the ray theory based signal representation can be considered as a special case of (1) with

$$
\mathcal{X}(\tau, \eta)=\sum_{i=1}^{N} a_{i} \delta\left(\tau-\tau_{i}\right) \delta\left(\eta-\eta_{i}\right),
$$

where $N$ is the number of ray paths, $a_{i}$ is the attenuation factor of the $i$ th ray, and $\delta(\cdot)$ is the Dirac delta function. As a result, in order to reduce the processing computational complexity at the receiver, we only need to estimate $N$ ray paths instead of all the WSF components.

The method we propose to estimate the ray paths is to first detect and separate the rays in the wideband ambiguity function (WAF) plane using a warping lag-Doppler filtering approach. Once the ray paths are separated, the corresponding attenuation coefficient for each ray path is estimated using a least-squares error (LSE) approach.

\section{B. Signal Warping}

Warping was considered as a way of nonlinearly mapping the time axis onto a new axis, where processing may be more easily applied [9]-[11]. Specifically, warping can be applied by transforming the time axis $t$ with a potentially nonlinear function $w(t)$. We introduce the linear and unitary warping operator $\mathcal{W}_{w}$ with associated warping function $w(t)$ such that when acting on a squareintegrable signal $g(t) \in \mathcal{L}^{2}(\mathbb{R})$, it transforms it as [12]

$$
\left(\mathcal{W}_{w} g\right)(t)=\left|\frac{d w(t)}{d t}\right|^{1 / 2} g(w(t))
$$

Let us consider $g(t)$ as a time-varying signal (i.e., as signal whose spectrum varies with time) that is monocomponent. Specifically, it can be given by

$$
g(t)=\mathrm{e}^{j 2 \pi c_{g} \varphi(t)},
$$


where $c_{g}$ is the frequency-modulation (FM) rate and $\varphi(t)$ is the time-varying (possibly nonlinear) phase function of the signal. For most realistic applications and analytical signals, we can assume that, given $\varphi(t)$, its derivative $\frac{d \varphi(t)}{d t}>0$, inverse function $\varphi^{-1}(t)$, and derivative of its inverse function $\frac{d \varphi^{-1}(t)}{d t}>0$ exist. Here, $\varphi\left(\varphi^{-1}(t)\right)=t$ for all $t$. Then, using the warped function $w(t)=\varphi^{-1}(t)$ in Equation (5), the projection of the signal $g(t)$ in Equation (6) in the warped time-domain defined by $\mathcal{W}_{\varphi^{-1}}$ yields an amplitude-modulated sinusoid. That is,

$$
\begin{aligned}
\left(\mathcal{W}_{\varphi^{-1}} g\right)(t) & =\left|\frac{d \varphi^{-1}(t)}{d t}\right|^{1 / 2} e^{j 2 \pi f_{g} \varphi\left(\varphi^{-1}(t)\right)} \\
& =\left|\frac{d \varphi^{-1}(t)}{d t}\right|^{1 / 2} e^{j 2 \pi f_{g} t}
\end{aligned}
$$

Equation (7) states that any signal $g(t)$ whose phase function is a known continuous and invertible function can be time-warped (using the inverse of the phase function of the signal) to warp the signal into a sinusoid. The resulting sinusoid can be accurately processed; for example, it can be time-frequency bandpass filtered. The filtering residue can be projected back in the initial time domain using the inverse warping operator $\mathcal{W}_{\varphi}$.

\section{Warping Lag-Doppler Filtering}

The aim of the warping lag-Doppler filtering (WALF) approach is to provide an efficient way of separating the different ray paths in the WAF plane [2], [7]. We define a dictionary of signals $\mathcal{D}$ that represent the timeshifted and scaled signals received after propagating over the wideband channel, as shown in Equation (1). The dictionary signals are given by

$$
g^{(m, n)}(t)=\sqrt{\eta_{m}} s\left(\eta_{m}\left(t-\tau_{n}\right)\right), \eta_{m} \neq 0,
$$

where $s(t)$ is the transmitted signal. The steps of the WALF iterative algorithm are summarized as follows.

We first initialize the process by defining $p_{0}(t)=x(t)$ where $x(t)$ is the received signal. Then, at the $i$ th iteration, $i=0,1, \cdots, M-1$, the projection of the residue $p_{i}(t)$ onto every dictionary element $g^{(m, n)}(t) \in \mathcal{D}$ is computed to obtain:

$$
\Lambda_{i}^{(m, n)}=\left\langle p_{i}, g^{(m, n)}\right\rangle \triangleq \int_{-\infty}^{+\infty} p_{i}(t) g^{(m, n) *}(t) d t .
$$

Then, we select the dictionary atom $g_{i}^{\left(m_{i}, n_{i}\right)}(t)$, with time-shift $\tau_{n_{i}}$ and scale $\eta_{m_{i}}$, which maximizes the magnitude of the projection:

$$
g_{i}^{\left(m_{i}, n_{i}\right)}(t)=\underset{g^{(m, n)}(t) \in \mathcal{D}}{\operatorname{argmax}}\left|\Lambda_{i}^{(m, n)}\right| .
$$

For realistic applications, we can assume that the phase function $\varphi(t)$ of $s(t)$ is differentiable and $\frac{d \varphi(t)}{d t}>0$. As $s(t)$ is assumed to be known, we assume that $\varphi(t)$ is known as well. We introduce $\varphi_{i}(t)$ to represent the phase function of $g_{i}^{\left(m_{i}, n_{i}\right)}(t)$ which is given by

$$
\varphi_{i}(t)=\varphi\left(\eta_{m_{i}}\left(t-\tau_{n_{i}}\right)\right) .
$$

The residue $p_{i}(t)$ is then warped with the time warping operator $\mathcal{W}_{\varphi_{i}^{-1}}$

$$
Q_{i}(t)=\left(\mathcal{W}_{\varphi_{i}^{-1}} p_{i}\right)(t) .
$$

As the warped signal is now a sinusoid, it can be filtered out easily in the warped time domain, as desired. According to the propagation properties, $\varphi_{j} \neq \varphi_{i}, \forall j \neq i$, $j=0,1, \cdots, M-1$. As a result, only the signal received for the $i$ th ray path is filtered out using a cut-band filter:

$$
U_{i}(t)=\mathcal{F} Q_{i}(t) .
$$

Here, $\mathcal{F}$ represents the cut-band filter that removes the narrowband function received for the $i$ th ray path after the WALF operation. The projection is then unwarped back to the time domain where the signal of the next WALF iteration is obtained as

$$
p_{i+1}(t)=\left(\mathcal{W}_{\varphi_{i}} U_{i}\right)(t) .
$$

\section{BASE07 EXPERIMENT}

The BASE07 experiment was jointly conducted by the NATO Undersea Research Center, the Forschungsanstalt der Bundeswehr für Wasserschall und Geophysik, the Applied Research Laboratory, and the Service Hydrographique et Océanographique de la Marine (SHOM). Two additional days of measurements were also conducted by SHOM to collect data for the geoacoustic inversion testing. Results based on the BASE07 experiment can be found in [2], [13], [14]. The real data we used was collected from a shallow water environment on the Malta Plateau. An linear, frequency-modulated (LFM) signal, whose spectrogram time-frequency representation is illustrated in Fig. 1, was transmitted by a source moving rectilinearly at constant speed from 2 to 12 knots and at different depths. The transmitted LFM signal had a $2 \mathrm{kHz}$ bandwidth, $1.3 \mathrm{kHz}$ central frequency, and a 4 $\mathrm{s}$ duration. Also, both the source and the receiver were moving with a relative velocity of $2.1 \mathrm{~m} / \mathrm{s}$, and the range between the transmitter and the receiver was $1,300 \mathrm{~m}$.

Figure 2 represents the WAF of the received signal where the crosses illustrate the ray path coordinates estimated with the WALF algorithm. The WAF values 


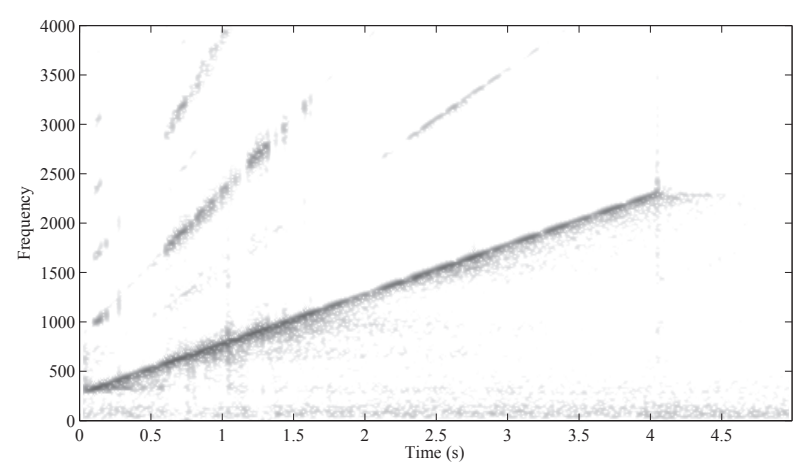

Fig. 1. Spectrogram of the LFM signal transmitted by the towed transmitter, demonstrating the effects of the multipath underwater propagation.

are computed during the processing as the values obtained with Equation (9) during the first WALF iteration. Figure 2 shows four ray paths that arrive at different time delays and different Doppler scaling factors. After a ray path is detected in the WAF plane, it is filtered out using the WALF approach. The advantage of our method is that the ray path detection provides an overall estimate of the ray Doppler scaling factor and time delay. As the channel is sparse, only a small dictionary was needed. Hence, for each ray path, the dictionary used for matrix inversion was small. In particular, only 4 possible scaling factors and 110 possible time delay factors, centered around the ray path estimates, were used for the matrix inversion. The global dictionary was much bigger as it was made of 19 possible scaling factors and 255 possible time delay factors. The attenuation coefficients (AC) in Equation (4), corresponding to the estimated WSF values for the sparse channel, obtained for each of the four detected ray paths as well as the ray path reconstructions from the estimated channel coefficients are presented in Figure 3. It is worth noting that for each ray path, the expected parameter ranges are very small and are around the center of the ray path. The ray path reconstruction errors are computed as follows:

$$
\epsilon=\frac{\int|x(t)-\hat{x}(t)|^{2} d t}{\int|x(t)|^{2} d t}
$$

where $x(t)$ is the original experimental data and $\hat{x}(t)$ is the recovered signal from the time-scale representation. The different reconstruction errors are $1.37 \%, 3.44 \%$, $7.86 \%$, and $5.86 \%$ for the first to the fourth ray path, respectively.

The global AC estimate is obtained from the estimates of the four ray paths. The global AC estimate is shown in

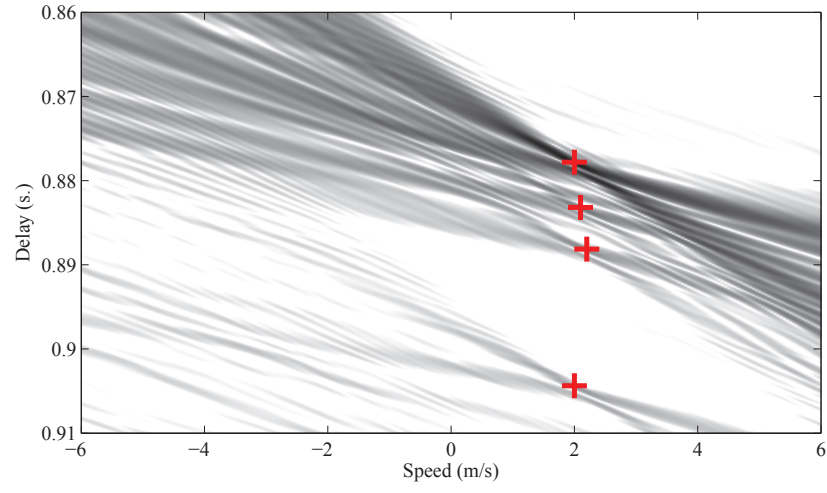

Fig. 2. WAF of a received signal. The crosses represent the position of the detected ray paths. Note that the speed parameter relates to the scale factor using the relationship $\eta=1 /(1-v / c)$, where $c$ is the speed of sound in the ocean.

Fig. 4; it enables the reconstruction of the signal received for the four ray paths with a reconstruction error of $3.4 \%$.

\section{CONCLUSION}

We introduced a new approach to characterize the signal propagating over wideband linear time-varying channels with a moving receiver and a moving transmitter. The method is based on the discrete time-scale characterization for acoustic signals and the WALF algorithm for extracting different arrival ray paths in order to decrease the computational complexity of the channel estimation when the channel is sparse. This method can be used for applications such as channel estimation for underwater acoustic communications, and active or passive tomography, when the relative transmitter-receiver velocity is unknown. The new approach was successfully evaluated using data from the BASE07 experiment.

\section{REFERENCES}

[1] L.H. Sibul, L.G. Weiss, and T.L. Dixon, "Characterization of stochastic propagation and scattering via Gabor and wavelet transforms," Journal of Computational Acoustics, vol. 2, no. 3, pp. 345-369, 1994.

[2] N.F. Josso, C. Ioana, J.I. Mars, C. Gervaise, and Y. Stéphan, "On the consideration of motion effects in the computation of impulse response for underwater acoustics inversion," Journal of the Acoustical Society of America, vol. 126, pp. 1739-1751, 2009.

[3] R.G. Shenoy and T.W. Park, "Wide-band ambiguity functions and affine Wigner distributions," EURASIP Journal on Signal Processing, vol. 41, pp. 339-363, 1995. 


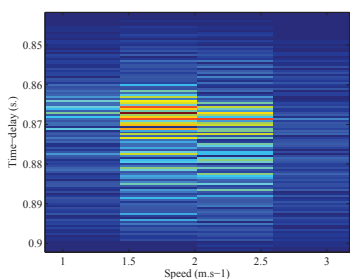

(a)

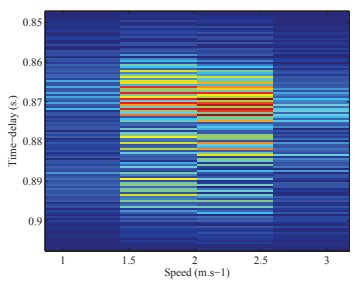

(c)

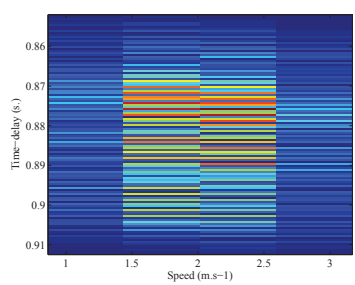

(e)

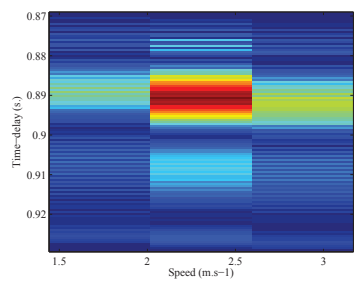

(g)

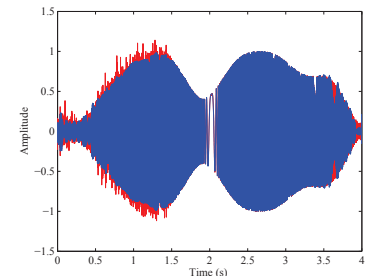

(b)

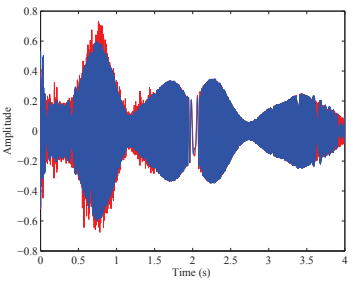

(d)

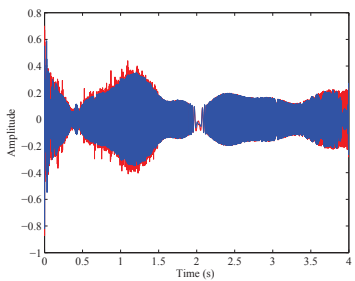

(f)

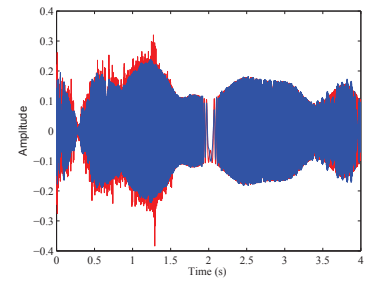

(h)
Fig. 3. AC of the: (a) first, (c) second, (e) third, and (g) forth forth ray group; recovered signal (blue) superimposed with the original signal for the: (b) first, (d) second, (f) third, and (h) forth ray group.

[4] B.G. Iem, A. Papandreou-Suppappola, and G.F. BoudreauxBartels, "Wideband Weyl symbols for dispersive time-varying processing of systems and random signals," IEEE Transactions on Signal Processing, vol. 50, pp. 1077-1090, May 2002.

[5] Y. Jiang and A. Papandreou-Suppappola, "Discrete time-scale characterization of wideband time-varying systems," IEEE Transactions on Signal Processing, vol. 54, no. 4, pp. 13641375, April 2006.

[6] A. Papandreou-Suppappola, C. Ioana, and J. Zhang, "Timescale and dispersive processing for time-varying channels," in Wireless Communications over Rapidly Time-Varying Channels. F. Hlawatsch and G. Matz, ed. Academic Press, 2009.

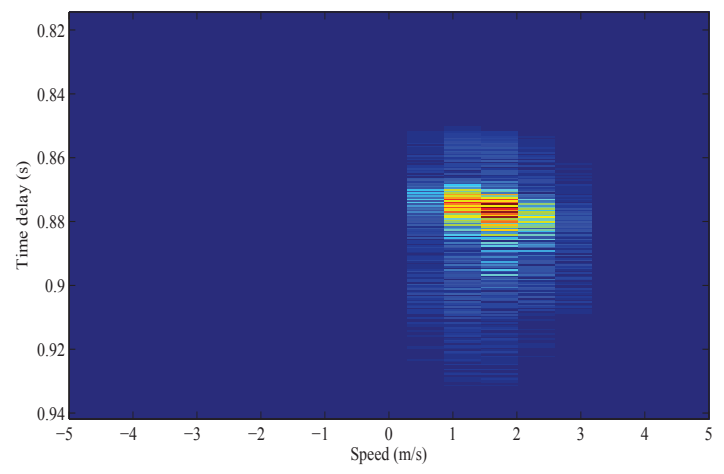

Fig. 4. Global AC estimate of the received signal.

[7] J.P. Hermand and W.I. Roderick, "Delay-Doppler resolution performance of large time-bandwidth-product linear FM signals in a multipath ocean environment," Journal of the Acoustical Society of America, vol. 84, pp. 1709-1727, 1988.

[8] J. Bertrand, P. Bertrand, and Ovarlez J.Ph., "The Melin transform," in The Tranforms and Applications Handbook. A. Poularikas, Ed. Boca Raton, FL: CRC, 1996.

[9] R.A. Altes, "Wide-band, proportional-bandwidth Wigner-Ville analysis," IEEE Transactions on Acoustics, Speech and Signal Processing, vol. 38, pp. 1005-1012, June 1990.

[10] A. Papandreou, F. Hlawatsch, and G. F. Boudreaux-Bartels, "The hyperbolic class of quadratic time-frequency representations Part I: Constant-Q warping, the hyperbolic paradigm, properties, and members," IEEE Transactions on Signal Processing, vol. 41, pp. 3425-3444, December 1993.

[11] R.G. Baraniuk and D.L. Jones, "Unitary equivalence: a new twist on signal processing," IEEE Transactions on Signal Processing, vol. 43, no. 10, pp. 2269-2282, Oct 1995.

[12] A. Jarrot, C. Ioana, and A. Quinquis, "Toward the use of the time warping principle with discrete time sequences," Journal of Computers, vol. 2, pp. 49-55, 2007.

[13] N.F. Josso, J.J. Zhang, A. Papandreou-Suppappola, C. Ioana, J.I. Mars, C. Gervaise, and Y. Stéphan, "On the characterization of time-scale underwater acoustic signals using matching pursuit decomposition," in OCEANS'09 MTS/IEEE Biloxi, Marine Technology for our Future: Global and Local Challenges, 2009.

[14] N. F. Josso, C. Ioana, C. Gervaise, Y. Stephan, and J. I. Mars, "Motion effect modeling in multipath configuration using warping based lag-Doppler filtering," IEEE International Conference on Acoustics, Speech, and Signal Processing, pp. 2301-2304, 2009. 